\title{
TORSION-FREE GROUPS ISOMORPHIC TO ALL OF THEIR NON-NILPOTENT SUBGROUPS
}

\author{
PATRIZIA LONGOBARDI, MERCEDE MAJ, HOWARD SMITH \\ and JAMES WIEGOLD
}

\author{
To Laci Kovács on his 65th birthday
}

(Received 29 September 2000)

Communicated by R. A. Bryce

\begin{abstract}
The main result is that every torsion-free locally nilpotent group that is isomorphic to each of its nonnilpotent subgroups is nilpotent, that is, a torsion-free locally nilpotent group $G$ that is not nilpotent has a non-nilpotent subgroup $H$ that is not isomorphic to $G$.
\end{abstract}

2000 Mathematics subject classification: primary $20 \mathrm{~F} 19$.

Keywords and phrases: torsion-free, locally nilpotent groups.

\section{Introduction}

One of the main results of [8], namely Theorem 1.1, is that a torsion-free soluble group $G$ that is isomorphic to each of its non-nilpotent subgroups is itself nilpotent if it is not finitely generated. On the other hand, if $G$ is finitely generated soluble and isomorphic to each of it non-nilpotent subgroups then either every proper subgroup of $G$ is nilpotent and hence, by [3, Lemma 3.2], $G$ is finite or else nilpotent, or $G$ satisfies the hypotheses of [7, Theorem 1]. Now if in addition $G$ is torsion-free then we deduce from this latter result that $G$ is isomorphic to each of its non-abelian subgroups, and now we may apply [6, Theorem 2]: again assuming that $G$ is not nilpotent we have that $G$ satisfies condition (vi) of that theorem, but the torsion-freeness of $G$ yields a contradiction. The above argument establishes the following.

The third author is grateful for the hospitality provided by the Department of Mathematics at the University of Napoli while part of this work was carried out.

(C) 2001 Australian Mathematical Society 0263-6115/2001 \$A2.00+0.00 
THEOREM 1. Let $G$ be a soluble group that is isomorphic to each of its non-nilpotent subgroups. If $G$ is torsion-free then $G$ is nilpotent.

The main result of the present work provides a generalization of [8, Theorem 1.1] in a different direction. We shall prove that the hypothesis of solubility is not in fact required-note that we cannot simultaneously remove the hypotheses of solubility and non-finite generation, as there exist (finitely generated) simple torsion-free groups with all proper subgroups cyclic (see [4]). Of course, a group that is isomorphic to each of its non-nilpotent subgroups is either finitely generated or locally nilpotent.

THEOREM 2. Let $G$ be a locally nilpotent group that is isomorphic to each of its non-nilpotent subgroups. If $G$ is torsion-free then $G$ is nilpotent.

Several of the results in [5] are concerned with torsion-free locally nilpotent groups $G$, and indicate that restrictions on the non-nilpotent subgroups of $G$ often imply nilpotency. Theorem 2 above is seen to be a result of this kind.

During the course of our discussion we shall frequently be applying some properties of isolators in (torsion-free) locally nilpotent groups. Firstly we recall the definition. If $G$ is a locally nilpotent group and $H$ is a subgroup of $G$ then the isolator of $H$ in $G$, denoted $I_{G}(H)$, is the set $\left\{g \in G: g^{n} \in H\right.$ for some positive integer $\left.n\right\}$. This is a subgroup of $G$, and the main properties that we shall require are as follows [1, Section 4]. Assume that $G$ is torsion-free, let $H$ be a subgroup of $G$, and let $\gamma_{i}(G)$ (respectively, $Z_{i}(G)$ ) denote the $i$ th term of the lower (respectively, upper) central series of the group $G$. If $I_{G}(H)=G$, then $C_{G}(H)=Z(G)$ and, for each positive integer $i, I_{G}\left(Z_{i}(H)\right)=Z_{i}(G)$ and $I_{G}\left(\gamma_{i}(H)\right)=I_{G}\left(\gamma_{i}(G)\right)$. If $H$ is nilpotent of class $c$, then so is $I_{G}(H)$. If $K$ is a normal subgroup of $H$, then $I_{G}(K)$ is normal in $I_{G}(H)$.

\section{Preliminary results}

In this section we present a few results that are required for the proof of Theorem 2. The first of these will in turn require a couple of lemmas.

PROPOSITION 1. Let $G$ be a torsion-free locally nilpotent group that is isomorphic to each of its non-nilpotent subgroups, and suppose that $G$ is not nilpotent. Then

(i) $G^{2}$ is a proper subgroup of $G$;

(ii) $G$ is a Fitting group; and

(iii) the hypercentre of $G$ is its centre.

LEMMA 1. Let $G$ be a countable torsion-free nilpotent group and suppose that for every subgroup $H$ of $G$ with $I_{G}(H)=G$ we have $G$ isomorphic to $H$. Then $G$ is abelian. 
PROOF. Suppose the result false and let $G$ be a counter-example of minimal nilpotency class $c$, say. Then $\bar{G}=G / Z_{c-2}(G)$ has class exactly 2 and, since $Z_{c-2}(G)=Z_{c-2}(H)$ for all $H$ satisfying $Z_{c-2}(G) \leq H$ and $I_{G}(H)=G$, we deduce that $\bar{G}$ is also a counter-example and hence that $c=2$.

Let $A=I_{G}\left(G^{\prime}\right)$ and let $K / A$ be a free abelian subgroup of $G / A$ such that $G / K$ is periodic. Then $K \simeq G$ and $I_{G}\left(K^{\prime}\right)=I_{G}\left(G^{\prime}\right)=A$. It follows that $I_{K}\left(K^{\prime}\right)=A$ and hence that $G / A \simeq K / A$, that is, $G / A$ is free abelian. Thus $G / G^{\prime}$ splits over $A / G^{\prime}$ and we have $G / G^{\prime}=H / G^{\prime} \times A / G^{\prime}$ for some free abelian subgroup $H / G^{\prime}$. In particular we have $G=H A$ and hence $G^{\prime}=H^{\prime}$ (since $A$ is central). Since $H / G^{\prime}$ is free abelian and $I_{G}(H)=G$, we deduce that $H / H^{\prime}$ is free abelian, as therefore is $G / G^{\prime}$. Write $G / G^{\prime}=\left\langle x_{1} G^{\prime}\right\rangle \times\left\langle x_{2} G^{\prime}\right\rangle \times \cdots$ for some (possibly finite) set $\left\{x_{1}, x_{2}, \ldots\right\}$.

Let $A_{1}=\left\langle\left[x_{1}, x_{2}\right]\right\rangle, I_{1}=I_{G^{\prime}}\left(A_{1}\right)$. There is a positive integer $n_{3}$ such that $\left[\left\langle x_{1}, x_{2}\right\rangle,\left\langle x_{3}^{n_{3}}\right\rangle\right] \cap I_{1} \leq A_{1}$, and hence $A_{1}\left[\left\langle x_{1}, x_{2}\right\rangle,\left\langle x_{3}^{n_{3}}\right\rangle\right]=A_{1} \times A_{2}$ for some (finitely generated) subgroup $A_{2}$. Let $I_{2}=I_{G^{\prime}}\left(A_{1} \times A_{2}\right)$ and choose $n_{4}>0$ with $\left[\left\langle x_{1}, x_{2}, x_{3}\right\rangle,\left\langle x_{4}^{n_{4}}\right\rangle\right] \cap I_{2} \leq A_{1} \times A_{2}$ and hence $\left(A_{1} \times A_{2}\right)\left[\left\langle x_{1}, x_{2}, x_{3}\right\rangle,\left\langle x_{4}^{n_{4}}\right\rangle\right]=A_{1} \times A_{2} \times$ $A_{3}$ for some subgroup $A_{3}$. Continue in this manner and set $X=\left\langle x_{1}, x_{2}, x_{3}^{n_{3}}, x_{4}^{n_{4}}, \ldots\right\rangle$. Clearly, $G^{\prime} I_{G}(X)=G_{\mathrm{b}}$ and so $I_{G}(X)=G$, since $G$ is nilpotent. By the choice of the $n_{i}, X^{\prime}$ is free abelian, as therefore is $G^{\prime}$ (since $X$ is isomorphic to $G$ ).

Now let $Y=X^{\prime} X^{p}$ for some fixed prime $p$, and note that $X$ is isomorphic to $Y$ since $I_{X}(Y)=X$, so that in particular $Y / Y^{\prime}$ is torsion-free. Let $a, b \in X$; then $[a, b]^{p^{2}}=\left[a^{p}, b^{p}\right] \in Y^{\prime}$, and so $[a, b]-\in Y^{\prime}$ and we deduce that $X^{\prime}=Y^{\prime}$. But $Y^{\prime}$ is generated by elements $\left[u^{p}, v^{p}\right]$, where $u, v \in X$ (again using that fact that $G$ is nil-2), and since $\left[u^{p}, v^{p}\right]=[u, v]^{p^{2}}$ we see that $Y^{\prime} \leq\left(X^{\prime}\right)^{p^{2}}$, which is a proper subgroup of $X^{\prime}$ since $X^{\prime}$ is free abelian. This contradiction completes the proof of Lemma 1 .

COROLLARY 1. Let $G$ be a group that satisfies the hypotheses of Proposition 1. Then $G / G^{\prime}$ is periodic.

Proof. Suppose the result false and let $I=I_{G}\left(G^{\prime}\right)$; then $G / I$ is torsion-free and nontrivial, and clearly $G$ is countable. By [8, Theorem 1.1], $G$ is not soluble and so $I$ is non-nilpotent and hence isomorphic to $G$. Now let $J=I_{G}\left(I^{\prime}\right)$; then $J=I_{I}\left(I^{\prime}\right)$ and so $I / J$ is isomorphic to $G / I$. Furthermore, $G / J$ is torsion-free and non-abelian, and $J$ is insoluble. Choose $x, y \in G$ such that $[x, y] \notin J$ and let $K=J\langle x, y\rangle$. Then $K / J$ is torsion-free nilpotent but not abelian, and it follows that $G$ itself has a torsion-free nilpotent image of class exactly two, so that if $N=I_{G}\left(\gamma_{3}(G)\right)$ then $G / N$ has class exactly two. Let $H$ be an arbitrary subgroup of $G$ that contains $N$ and satisfies $I_{G}(H)=G$. If $\theta$ is an isomorphism from $G$ to $H$ then, since $I_{H}\left(\gamma_{3}(H)\right)$ is also $N$, we have that $N \theta=N$ and hence that $G / N$ is isomorphic to $H / N$. But now $G / N$ is a group that satisfies the hypotheses of Lemma 1 , and we obtain the contradiction that $G / N$ is abelian. 
The next result states a little more than is necessary for the proof of Proposition 1 but will be required in its more general form later.

LEMMA 2. Let $G$ be a locally nilpotent group, $N$ a normal torsion-free subgroup of $G$, and suppose that $N / M$ is periodic for every nontrivial $G$-invariant subgroup $M$ of $N$. Then $N$ is central in $G$ (and hence of rank one).

Proof. First we show that $N$ is abelian. Assuming this to be false, choose noncommuting elements $a, b$ of $N$ and set $c=[a, b]$. Then $I_{N}\left(\langle c\rangle^{G}\right)=N$ and so there is a finitely generated subgroup $F$ of $G$ with $a, b \in F$ and $a^{m}, b^{n} \in\langle c\rangle^{F}$ for some positive integers $m$ and $n$. Write $U=\langle a, b\rangle^{F}, V=\langle c\rangle^{F}$; then $U$ is generated by conjugates of $a$ and $b$ in $F$ and so the isolator of $V$ in $U$ is $U$ and hence $U / V$ is periodic. Since $V \leq U^{\prime}$ we therefore have $U / U^{\prime}$ periodic and hence $U$ periodic, since it is nilpotent. But $N$ is torsion-free and so we obtain the contradiction that $U$ is trivial, and it follows that $N$ is abelian.

Assume now that $N$ is not contained in $Z(G)$ and choose $g \in G$ that does not centralize $N$. Let $a$ this time be some element of $N$ with $[a, g]$ nontrivial, and set $b=[a, g]$. Since $N /\langle b\rangle^{G}$ is periodic we have $a^{m} \in\langle b\rangle^{F}$ for some finitely generated subgroup $F$ of $G$ and positive integer $m$. Let $H=\left\langle a^{m}, g, F\right\rangle$, a finitely generated and hence nilpotent subgroup of $G$, and let $A=\left\langle a^{m}\right\rangle^{H}$, which is abelian and normal in $H$. Since $N$ is abelian and $\langle g\rangle$-invariant, we see that $b^{m}=[a, g]^{m}=\left[a^{m}, g\right] \in[A, H]$. So, for every $h \in H,\left(b^{m}\right)^{h}$ is contained in $[A, H]$, and thus $\left\langle b^{m}\right\rangle^{F} \leq[A, H]$, which in turn gives $a^{m^{2}} \in\left\langle b^{m}\right\rangle^{F} \leq[A, H]$ and hence $\left\langle a^{m^{2}}\right\rangle^{H} \leq[A, H]$. It follows that $A /[A, H]$ is periodic and hence that $A /\left[A,{ }_{i} H\right]$ is periodic for each positive integer $i$. But $H$ is nilpotent and we deduce that $A$ is periodic and hence trivial, giving the contradiction that $b=1$. This concludes the proof of the lemma.

ProOF OF PROPOSITION 1. (i) Let $g$ be a nontrivial element of $G$. Certainly $\{g\} \cap$ $\left\langle g^{2}\right\rangle=\emptyset$, and so we may apply [2, Lemma 2] to obtain a subgroup $H$ of $G$ such that $\left\langle g^{2}\right\rangle \leq H, g \notin H$ and $I_{G}(H)=G$. Since $H$ is not nilpotent we have $G$ isomorphic to $\langle H, g\rangle$. Let $K$ be a subgroup of $\langle H, g\rangle$ that is maximal with respect to containing $H$ but not $g$; clearly $K$ is a maximal subgroup of index 2 in $\langle H, g\rangle$, and (i) follows.

(ii) Let $K$ denote the Fitting subgroup of $G$ and suppose that $K \neq G$. Since $K$ is of course a Fitting group it is not isomorphic to $G$ and is therefore nilpotent. It follows that $K$ is the unique maximal normal nilpotent subgroup of $G$, and since the isolator of $K$ is also nilpotent we have $G / K$ torsion-free and non-trivial. By [8, Theorem 1.1] $G$ is not soluble, and we may apply Lemma 3 to obtain a nontrivial normal subgroup $M / K$ of $G / K$ with $G / M$ not periodic. Replacing $M$ by its isolator (if necessary) we may assume that $G / M$ is torsion-free. Let $x \in G \backslash M$ and consider the subgroup $H=M(x)$; we see that $H$ is isomorphic to $G$ and that $H / M$ is infinite cyclic, and Corollary 1 gives a contradiction. Thus (ii) is established. 
(iii) If the hypercentre of $G$ is not $Z(G)$ then we may choose an element $x$ of $Z_{2}(G) \backslash Z(G)$ and consider the map $G \rightarrow Z(G)$ given by $g \rightarrow[g, x]$ for all $g \in G$. This is a homomorphism with nontrivial torsion-free abelian image, and Corollary 1 gives us another contradiction.

Our next requirement is as follows.

PROPOSITION 2. Let $G$ be a torsion-free locally nilpotent group that is isomorphic to each of its non-nilpotent subgroups and let $H$ be a non-nilpotent subgroup of $G$. Then $I_{G}(H)=G$.

ProOF. Assuming the result false, there exists a non-nilpotent subgroup $H$ of $G$ and a nontrivial element $g$ of $G$ with $H \cap\langle g\rangle=1$; clearly we may assume that $G=\langle H, g\rangle$. Let $N=\langle g\rangle^{C}$, which is nilpotent by Proposition 1 (ii), and note that $G=H N$. Let $L$ be an $H$-invariant subgroup of $N$ maximal with respect to containing $N \cap H$ and intersecting $\langle g\rangle$ trivially. Also, let $i$ be maximal such that $Z_{i}=Z_{i}(N)$ is contained in $L$, so that $Z_{i} \leq L$ but $Z_{i+1} \not L L$. There is a positive integer $n$ such that $g^{n} \in L Z_{i+1}$; if $g^{k} \in H L$ for some $k>0$ then $g^{k} \in H L \cap N=L(H \cap N)=L$, and we have a contradiction. Thus $\langle g\rangle \cap H L=1$. Since $g^{n} \in L Z_{i+1}$ we have $\left[L,\left\langle g^{n}\right\rangle\right] \leq\left[L, L Z_{i+1}\right] \leq L Z_{i}=L$. Thus $L \triangleleft\left\langle L, g^{n}\right\rangle$ and $L \triangleleft J=\left\langle H, L, g^{n}\right\rangle$; however, $\left\langle g^{n}\right\rangle \cap H L=1$. We shall prove that $H L$ is normal in $J$ - it will follow that $J=(H L) \rtimes\left\langle g^{n}\right\rangle$ and $J$ is isomorphic to $G$, contradicting the fact that $G / G^{\prime}$ is periodic (Corollary 1 ).

We know from the definition of $L$ that every $H$-invariant subgroup $M$ of $\left\langle g^{n}\right\rangle^{H} L$ that properly contains $L$ also contains a non-zero power of $g$. Thus $\left\langle g^{n}\right\rangle^{H} L / M$ is periodic for all such $M$. Certainly, therefore, every $J$-invariant subgroup $M$ of $\left\langle g^{n}\right\rangle^{H} L$ that properly contains $L$ has this property. Since $\langle g\rangle \cap L$ is trivial, $\left\langle g^{n}\right\rangle^{H} L / L$ is not periodic, so its torsion subgroup is trivial (else we may choose $M / L$ to be its torsion subgroup in the above). Now $\left\langle g^{n}\right\rangle^{H} L=\left\langle g^{n}\right\rangle^{J} L$, which is normal in $J$. So the normal torsion-free subgroup $\left\langle g^{n}\right\rangle^{J} L / L$ of $J / L$ has the property described in Lemma 3, and it follows that $\left\langle g^{n}\right\rangle^{J} L / L$ is central in $J / L$ and hence, in particular, that $H L$ is normalized by $\left\langle g^{n}\right\rangle$ and therefore normal in $J$. As we have seen, this establishes the result.

We know from Proposition 1 that a group $G$ that satisfies the hypotheses of our theorem is a Fitting group. The next result shows that if $G$ is not nilpotent then it is not generated by normal nilpotent subgroups of bounded class (the requirement that $G$ have trivial centre being a minor restriction, as we shall see).

PROPOSITION 3. Let $G$ be a torsion-free locally nilpotent group that is isomorphic to each of its non-nilpotent subgroups, and suppose that the centre of $G$ is trivial. Let 
$c$ be an arbitrary positive integer and let $N_{c}$ be the subgroup generated by all normal subgroups of $G$ that are nilpotent of class at most $c$. Then $N_{c}$ is nilpotent and, if $H$ is a non-nilpotent subgroup of $G$ and $\varphi$ is an isomorphism from $G$ to $H$, then $\varphi\left(N_{c}\right) \leq N_{c}$. Furthermore, if $S_{c}$ denotes the isolator of $N_{c}$ in $G$ then $G / S_{c}$ is torsion-free locally nilpotent and isomorphic to each of its non-nilpotent subgroups.

The main step in the proof of this result is provided by the following.

\section{LEMMA 3. The result of Proposition 3 holds in the case $c=1$.}

ProOF. Let $G$ be as given in the statement of the proposition and write $N=N_{1}$, $S=I(N)$, where all isolators here are isolators in $G$. Suppose we have shown that $N$ is nilpotent, so that $S$ is also nilpotent (and certainly $G / S$ is torsion-free). Let $H$ and $\varphi$ be as stated and let $A$ be a normal abelian subgroup of $G$. Then $\varphi(A)$ is a normal abelian subgroup of $H$ and $I(\varphi(A))$ is abelian and normal in $I(H)$, which equals $G$ by Proposition 2. It follows that $I(\varphi(A)) \leq N$ and hence $\varphi(A) \leq N$, and since $A$ was arbitrary we have $\varphi(N) \leq N$. Next, if $H / S$ is a non-nilpotent subgroup of $G / S$ then there is an isomorphism $\theta$ from $G$ to $H$, and by the above $\theta(N)$ is contained in $N$. Also, if $A$ is a normal abelian subgroup-of $G$ then $A$ is normal in $H$ and hence contained in $\theta(N)$; it follows that $\theta(N)=N$, and it is easy to see that $\theta(S)=S$. Thus $\theta$ induces an isomorphism from $G / S$ to $H / S$, and we are done.

It remains to show that $N$ is nilpotent, and we assume for a contradiction that this is not the case, so that $N$ is isomorphic to $G$ and hence $N$ equals $G$. Now $G / G^{\prime}$ is periodic, by Corollary 1 , and as in the proof of Proposition 1 (iii) it follows that, for every torsion-free image $K$ of $G$, the centre of $K$ is its hypercentre. We write $G=\left\langle A_{i}: A_{i}\right.$ is abelian, normal and isolated in $\left.G\right\rangle$, where $i$ runs through some index set $J$. Choose a nontrivial element $x$ of $G$.

We proceed to construct a sequence $B_{1}, B_{2}, \ldots$ of subgroups from among the $A_{i}$ such that, for each positive integer $n$, the following properties hold.

(i) $x \notin Z\left(G / I\left(B_{1} \cdots B_{n}\right)\right)$ (that is, $x$ is not central modulo $I\left(B_{1} \cdots B_{n}\right)$ ).

(ii) the nilpotency class $c_{n}$ of $B_{1} \cdots B_{n}$ exceeds that of $B_{1} \cdots B_{n-1}$ (interpreted as 0 in the case $n=1$ ).

Suppose first that $x \in Z\left(G / I\left(A_{i}\right)\right)$ for all $i \in J$ and let $g \in G$. Then $[x, g] \in I\left(A_{i}\right)$ and hence $[x, g]$ centralizes $A_{i}$ for all $i$, so that $[x, g] \in Z(G)=1$ and $x \in Z(G)$, a contradiction. Thus we may choose $B_{1}$, so that (i) and (ii) hold. Now assume that, for some $n$, we have found subgroups $B_{1}, \ldots, B_{n}$ among the $A_{i}$ so that (i) and (ii) hold, and write $G_{1}=\left\langle A_{j}: x \in Z\left(G / I\left(B_{1} \cdots B_{n} A_{j}\right)\right)\right\rangle, G_{2}=\left\langle A_{j}: x \notin\right.$ $\left.Z\left(G / I\left(B_{1} \cdots B_{n} A_{j}\right)\right)\right\rangle$. Then $G_{1}$ and $G_{2}$ are both normal in $G$, and $G=G_{1} G_{2}$, so at least one of $G_{1}$ and $G_{2}$ is non-nilpotent and hence $G=I\left(G_{1}\right)$ or $G=I\left(G_{2}\right)$, by Proposition 2. Write $L=I\left(B_{1} \cdots B_{n}\right)$ and let $g \in G$. Then $[x, g]$ centralizes $G_{1} \bmod$ 
$L$ and so, if $G=I\left(G_{1}\right)$, we see that $[x, g] \in Z(G / L)$, which gives $x \in Z_{2}(G / L)$ (that is, $[x, G, G] \leq L)$ and hence $x \in Z(G / L)$, contradicting (i). Hence $G=I\left(G_{2}\right)$. With the obvious notation, write $G_{2}=\left\langle A_{j}: j \in J^{*}\right\rangle$. If $B_{1} \cdots B_{n} A_{j}$ has nilpotency class $c_{n}$ (see (ii) above) for all $j \in J^{*}$ then we choose a nontrivial element $y$ of $\gamma_{c_{n}}\left(B_{1} \cdots B_{n}\right)$ and note that $y$ centralizes each such $A_{j}$ and hence centralizes $G_{2}$, and we obtain the contradiction $y \in Z(G)$. Thus there exists $j \in J^{*}$ such that the class $c_{n+1}$ of $B_{1} \cdots B_{n} A_{j}$ is greater than $c_{n}$, and we set $B_{n+1}=A_{j}$. The existence of our (infinite) sequence $B_{1}, B_{2}, \ldots$ is thus established by induction.

Now let $B=\left\langle B_{i}: i=1,2, \ldots\right\rangle$. By (ii) above $B$ is not nilpotent and so $I(B)=G$, which implies that $x^{k} \in B_{1} \cdots B_{n}$ for some positive integers $k$ and $n$, and hence that $x \in I\left(B_{1} \cdots B_{n}\right)$, a contradiction that completes the proof of the lemma.

PROOF OF PROPOSITION 3. We show that $N_{c}$ is nilpotent for each $c \in \mathbb{N}$; the remainder of the statement of the proposition follows just as for the case where $c=1$ (in the proof of Lemma 4). Assuming the result false, let $c$ be least such that $N_{c}$ is not nilpotent, so $c>1$ by Lemma 4 and $N_{c-1}$ is nilpotent, and $G / S_{c-1}$ is torsion-free and isomorphic to each of its non-nilpotent subgroups. Let $M / S_{c-1}$ be the centre of $G / S_{c-1}$. Then $G / M$ is also torsion-free and isomorphic to each of its non-nilpotent subgroups, since for every non-nilpotent subgroup $H / M$ of $G / M$ we have $I(H)=G$ (by Proposition 2), and hence $M / S_{c-1}=Z\left(H / S_{c-1}\right)$, so that $M$ is invariant under any isomorphism from $G$ to $H$ (since $S_{c-1}$ is thus invariant). If $K$ is an arbitrary normal nilpotent subgroup of $G$ of class at most $c$ then $K^{\prime} \leq N_{c-1} \leq M$, and it follows that $N_{c}$ is generated modulo $M$ by normal abelian subgroups of $G$. Applying Lemma 4 (and part (iii) of Proposition 1) to the group $G / M$ we deduce that $N_{c} M / M$ is nilpotent. But $M$ is soluble and therefore so is $N_{c}$, and [8, Theorem 1.1] gives the contradiction that $N_{c}$ is nilpotent. Thus Proposition 3 is proved.

\section{Proof of Theorem 2}

Suppose that $G$ is a torsion-free locally nilpotent group isomorphic to each of its non-nilpotent subgroups and, for a contradiction, that $G$ is not nilpotent. Let $Z=Z(G)$ and note that if $H / Z$ is a non-nilpotent subgroup of $G / Z$, then $I_{G}(H)=G$ by Proposition 2 and so $Z=Z(H)$. It follows that $G / Z$ satisfies the hypotheses of the theorem and so, by Proposition 1 (iii), we may factor by $Z$ and hence assume that $G$ has trivial centre. For each positive integer $k$, let $N_{k}=\langle A: A \triangleleft G$ and $A$ is nilpotent of class at most $k\rangle$. If $N_{k} \leq G^{2}$ for all $k$ then, since $G$ is a Fitting group (Proposition 1 (ii)), $G=G^{2}$, contradicting Proposition 1 (i). Thus there exists an integer $m$ such that $N_{m} \not \leq G^{2}$. Write $S_{m}=I_{G}\left(N_{m}\right)$; then, by Proposition 3, $S_{m}$ is nilpotent and $G / S_{m}$ is torsion-free and isomorphic to each of its non-nilpotent subgroups. By Corollary 1 we may write $G / G^{2}=G^{2} S_{m} / G^{2} \times B / G^{2}$, where $B / G^{2}$ is nontrivial. 
We construct, inductively, a sequence $\left\{b_{1}, b_{2}, \ldots\right\}$ of elements of $B$ such that, for each positive integer $n,\left[b_{1}, \ldots, b_{n}\right] \neq 1$ and $b_{1}, \ldots, b_{n}$ are linearly independent $\bmod G^{2}$. Choose a nontrivial element $b_{1}$ of $B$. Suppose that $\left[b_{1}, \ldots, b_{n}\right] \neq 1$, where $b_{1}, \ldots, b_{n}$ are linearly independent $\bmod G^{2}$, and let $D=\left\langle b_{1}, \ldots, b_{n}\right\rangle^{G}$. By Proposition 1 (ii) $D$ is nilpotent and therefore contained in $N_{c}$ for some integer $c$. Assuming as we may that $c \geq m$, and writing $I=I_{G}\left(N_{c}\right)$, we note from Proposition 3 that $G / I$ is torsion-free non-nilpotent and isomorphic to each of its non-nilpotent subgroups. By Corollary 1 we have $G^{2} I<G$ and hence $G^{2}(B \cap I)<B$ (else $B \leq G^{2} I$ and $G=G^{2} I$, a contradiction). Thus $B / G^{2}=G^{2}(B \cap I) / G^{2} \times C / G^{2}$ for some $C$ not contained in $G^{2}$. If $C$ is nilpotent then so is its isolator $G$, a contradiction, and it follows that $C$ is isomorphic to $G$. Now if $\left[b_{1}, \ldots, b_{n}, c\right]=1$ for all $c \in C \backslash G^{2}$ then, $\left[b_{1}, \ldots, b_{n}\right] \in Z(G)=1$, since $C$ is generated by all such $c$ and $I_{G}(C)=G$. By this contradiction there exists $b_{n+1} \in C \backslash G^{2}$ with $\left[b_{1}, \ldots, b_{n+1}\right] \neq 1$, and since $b_{1}, \ldots, b_{n+1}$ are linearly independent $\bmod G^{2}$ the claim is established.

Now write $H=\left\langle b_{n}: n \in \mathbb{N}\right\rangle$; then $H$ is non-nilpotent and so there is an isomorphism $\varphi$ from $G$ to $H$. By Proposition 3, $\varphi\left(N_{m}\right) \leq N_{m}$ and hence $\varphi\left(N_{m}\right) \leq S_{m} \cap B \leq$ $G^{2}$, so $\varphi\left(N_{m}\right) \leq G^{2} \cap H$. For each $a \in \varphi\left(N_{m}\right)$ we have $a H^{2}=b_{1}^{\alpha_{1}} \cdots b_{n}^{\alpha_{n}} H^{2}$ for some $n$, where each $\alpha_{i}=0$ or 1 , and since $\varphi\left(N_{m}\right) \leq G^{2}$ it follows that $a H^{2} \subseteq G^{2}$ and hence that each $\alpha_{i}=0$, so that $a \in H^{2}$ and $\varphi\left(N_{m}\right) \leq H^{2}$. Thus $N_{m} \leq G^{2}$, a contradiction that completes the proof of the theorem.

\section{References}

[1] P. Hall, Nilpotent groups, Collected works of Philip Hall (Clarendon Press, Oxford, 1988).

[2] W. Möhres, 'Torsionsfreie Gruppen, deren Untergruppen alle subnormal sind', Math. Ann. 284 (1989), 245-249.

[3] M. F Newman and J. Wiegold, 'Groups with many nilpotent subgroups', Arch. Math. 15 (1964), 241-250.

[4] A. Yu. Ol'shanskii, Geometry of defining relations in groups (Nauka, Moscow, 1989).

[5] H. Smith, 'Groups with few non-nilpotent subgroups', Glasgow Math. J. 39 (1997), 141-151.

[6] H. Smith and J. Wiegold, 'Groups which are isomorphic to their nonabelian subgroups', Rend. Sem. Mat. Univ. Padova 97 (1997), 7-16.

[7] _- 'Groups isomorphic to their non-nilpotent subgroups', Glasgow Math. J. 40 (1998), 257-262.

[8] _ , 'Soluble groups isomorphic to their non-nilpotent subgroups', J. Austral. Math. Soc. (Series A) 67 (1999), 399-411.

Dipartimento di Matematica e Informatica via $S$. Allende Bucknell University 84081 Baronissi Lewisburg PA 17837 Italy USA e-mail: longobar@matna2.dma.unina.it e-mail: maj@matna2.dma.unina.it 
School of Mathematics

Cardiff University

Cardiff CF24 4Y

Wales

e-mail: smajw@cardiff.ac.uk 
